\title{
New Insights in Pharmaceutical Analysis
}

\author{
Davy Guillarme, Julie Schappler, Julien Boccard, Jean-Luc Veuthey, and Serge Rudaz*
}

\begin{abstract}
The research unit of pharmaceutical analytical chemistry (PAC) has been active in the field of separation sciences for many years. Liquid chromatography (LC) and its latest improvements such as ultra-high performance chromatography (UHPLC) and supercritical fluid chromatography (SFC) are deeply and thoroughly studied, from a fundamental viewpoint to its various application capabilities. Electro-driven separations such as capillary electrophoresis (CE) are also a major field of interest, especially for macromolecules, and low cost. All these techniques are investigated with various detection modes including mass spectrometry (MS) for various applications where high sensitivity and selectivity is needed. Extracting the relevant information from the overwhelming amount of data generated by modern analytical platforms has become an important issue for knowledge discovery in various research fields. The appropriate treatment of such data is therefore of crucial importance to provide valuable information. Numerous works in our research group have demonstrated the usefulness of statistical and mathematical methodologies to improve quality of the results. Therefore, well-established chemometric approaches (e.g. design of experiments, multivariate data analysis, etc.) are implemented to optimize the analytical process from method development to data analysis.
\end{abstract}

Keywords: Capillary electrophoresis - Chemometrics - Fast analysis - Liquid chromatography . Mass spectrometry · Metabolism · Metabolomics · Toxicological analysis

\section{Introduction}

For the past 20 years (1992-2012), the research unit of pharmaceutical analytical chemistry (PAC) has been working on developing rapid and high-resolution methods for pharmaceutical analysis using liquid chromatography (LC/UHPLC), supercritical fluid chromatography (SFC) and capillary electrophoresis (CE) combined with various detectors, including mass spectrometry (MS). Fundamental aspects and new strategies in separation sciences have been studied to gain resolution, selectivity and/or sensitivity for low molecular weight analytes, peptides and proteins, including sample preparation techniques. Particular attention has been paid to methodology related to the acquisition of experimental results and data treatment. Chemometric tools, such as design

\footnotetext{
${ }^{*}$ Correspondence: Dr. S. Rudaz

Research Unit of Pharmaceutical Analytical Chemistry School of Pharmaceutical Sciences

Geneva-Lausanne

University of Geneva, 20 bd d'Yvoy

$\mathrm{CH}-1211$ Geneva 4

Tel.: +4122379 6572

Fax: +412237968 08

E-mail: serge.rudaz@unige.ch
}

of experiments (DoE) and advanced multivariate analysis (MVA), have been used in metabolomics, metabolism studies and applied human toxicology. This paper describes the most recent projects performed in collaboration with industrial, academic or institutional partners.

\section{Liquid Chromatography (LC)}

In the pharmaceutical field, liquid chromatography (LC) is used extensively for the qualitative as well as the quantitative analysis of active substances. During the last five years, there has been a consistent trend towards improving the speed of analysis, obtaining higher resolving power, enhancing selectivity or performing greener analyses. These goals can generally be attained only with the latest generation of instruments and columns.

An initial strategy consists of using columns packed with sub- $2 \mu \mathrm{m}$, fully porous particles with chromatographic systems able to withstand pressures of up to 1300 bars. This strategy was described theoretically and applied to solve numerous problems in our research group. Chromatography under such extreme conditions is generally described as UHPLC (ultra-high pressure liquid chromatography) and produces either fast separations or high resolution. Another benefit of UHPLC is the possibility of direct transfer of existing HPLC methods. A calculator freeware program based on fundamentals in chromatography and available on our website ${ }^{[1]}$ was developed to determine the new conditions for both isocratic and gradient modes.

The ultra-high resolution profiling of complex samples such as plant extracts was also developed in UHPLC, with relatively long analyses (>100 $\mathrm{min})$ and a mobile phase temperature of $90{ }^{\circ} \mathrm{C} .[2,3]$ These conditions allowed the separation of more than 400 constituents. As a part of a collaboration with the Swiss Laboratory for Doping Analyses (LAD, Lausanne, Switzerland), a methodology based on UHPLC coupled with quadrupole time-offlight mass spectrometry (QTOF-MS) was developed to screen and confirm 103 doping agents from various classes. ${ }^{[4,5]}$

A limitation of the UHPLC strategy is related to the heat induced by friction when columns packed with very fine particles are used at high mobile phase velocities, thus generating significant pressure drop. ${ }^{[6]}$ To avoid frictional heating, it is now possible to use alternative strategies, such as columns packed with sub- $3 \mu \mathrm{m}$ core-shell particles. The core-shell particles consist of a $1.7 \mu \mathrm{m}$ solid inner core surrounded by a $0.5 \mu \mathrm{m}$ porous layer. This type of column generates significantly less backpressure and provides chromatographic performance that is almost equivalent to the fully porous sub- $2 \mu \mathrm{m}$ columns employed in UHPLC. ${ }^{[7]} \mathrm{A}$ recent study dealing with real analysis of simple mixtures of drugs also confirmed the potential of this approach as the core-shell column provided kinetic performance, retention, and selectivity, as well as loading capacity, similar to conventional UHPLC columns. ${ }^{[8]}$

Peptides and proteins are currently 


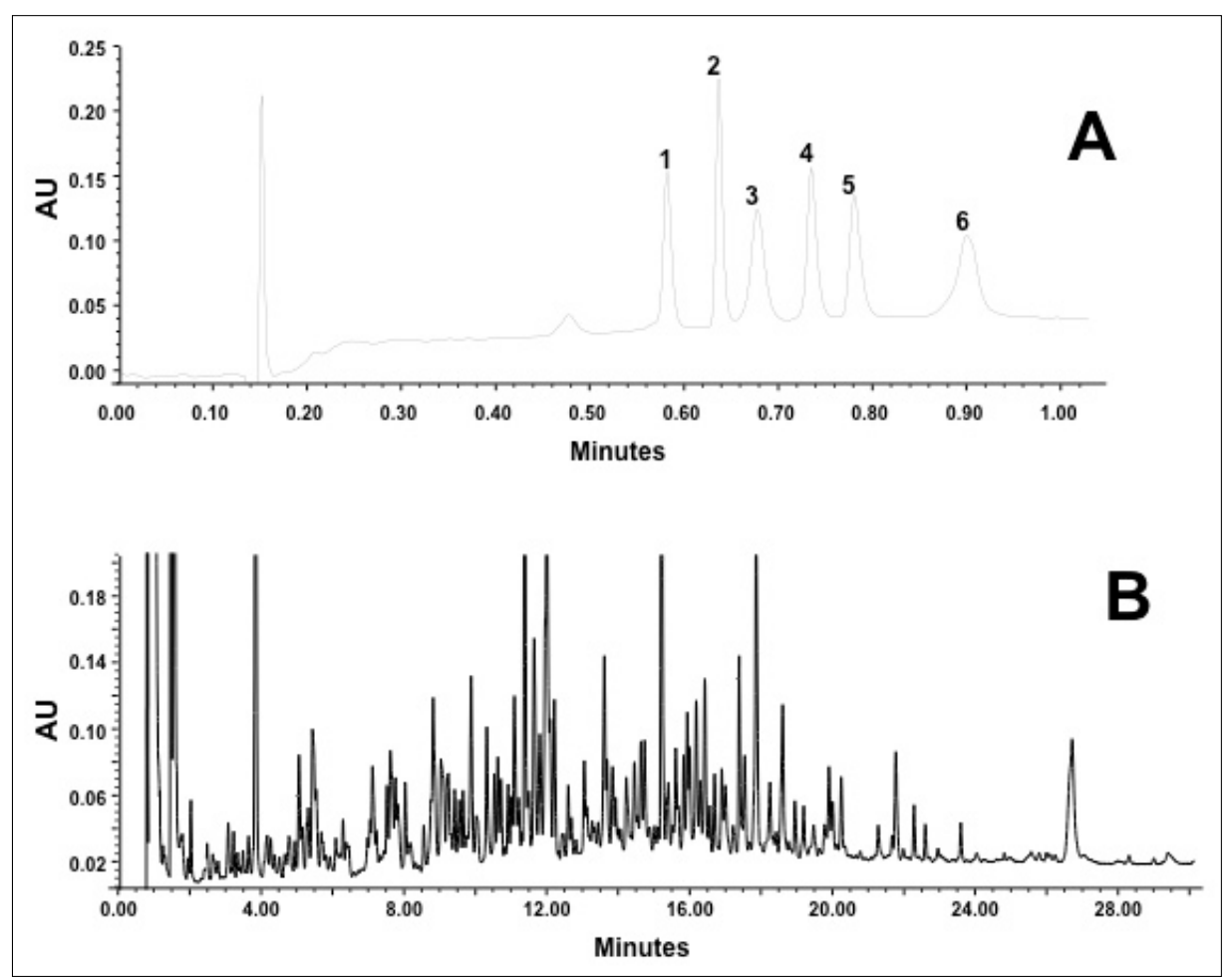

Fig. 1. A. Fast separation of model proteins: 1. cytochrome $C(50 \mu \mathrm{g} / \mathrm{mL})$, 2. insulin $(100 \mu \mathrm{g} / \mathrm{mL})$, 3. $\alpha$-glycoprotein acid $(100 \mu \mathrm{g} / \mathrm{mL})$, 4. $\alpha$-lactalbumin $(50 \mu \mathrm{g} / \mathrm{mL}), 5$. myoglobin $(60 \mu \mathrm{g} / \mathrm{mL})$, and 6 . ovalbumin $(200 \mu \mathrm{g} / \mathrm{mL})$. B. High-resolution tryptic digest profiling of a protein mixture: ovalbumin, $\beta$-lactoglobulin, human serum albumin, and myoglobin. Adapted from ref. [9] with permission.

recognized as new therapeutic agents. Dedicated analytical methodologies are also developed. Because of its high resolving power and inherent compatibility with MS, reversed-phase LC (RPLC) is of utmost interest. The applicability of sub-2 $\mu \mathrm{m}$ porous and sub-3 $\mu \mathrm{m}$ core-shell particles was demonstrated in this context. The separation power and throughput were quite elevated, as demonstrated in Fig. 1, with ultra-fast separation of various intact proteins in less than $1 \mathrm{~min}$ (Fig. 1A) and the high resolution separation of several digested proteins in approximately $30 \mathrm{~min}$ utes (Fig. 1B). ${ }^{[8]}$

Another topic of interest for any separation scientist is achieving selectivity with complex samples and attaining sufficient retention of very polar analytes. These two goals can be achieved with the hydrophilic interaction liquid chromatography (HILIC) mode in which the stationary phase is polar (bare silica or polar bonding) and the mobile phase is a mixture of aprotic organic solvent (e.g. acetonitrile) and aqueous buffer. This chromatographic mode was described at the beginning of the 90 s but has only recently found widespread application and is currently under investigation. The concept of green analytical chemistry has recently gained increased attention from the scientific community because of the rising costs associated with the use and reprocessing of the large volumes of organic solvents generated by HPLC. UHPLC is attractive, as the application of this technique allows an approximately 14-fold decrease in organic solvent consumption compared with conventional HPLC for the same number of analyses. Supercritical fluid chromatography (SFC) provides an alternative choice to HPLC because the main organic eluent is replaced by pressurized $\mathrm{CO}_{2}$ containing only a limited amount of organic solvent modifier (5-30\%). Some significant improvements in terms of SFC instrumentation and columns have recently been observed, particularly with the launch of new SFC columns packed with sub- $2 \mu \mathrm{m}$ particles. We are currently investigating the possibilities offered by SFC with conventional as well as sub- $2 \mu \mathrm{m}$ columns for the analysis of drugs.

\section{Capillary Electrophoresis (CE)}

Capillary electrophoresis (CE) is a powerful separation technique and has found numerous applications in various analytical fields. Several advantages, such as high efficiency, rapid method development, simple instrumentation and low sample consumption, are the main reasons for the success of CE. UV-VIS spectrophotometry is probably the detection technique most widely used with $\mathrm{CE}$ because of the simplicity of the on-line configuration. However, the sensitivity of the UVVIS detector, directly related to the optical pathlength afforded by the internal diameter of the capillaries (in the $\mu \mathrm{m}$ range), remains the major bottleneck to the use of this technique. Other systems are therefore required for the analysis of complex mixtures, and several alternatives, including laser-induced fluorescence, conductivity, and mass spectrometry (MS) detection techniques, have been investigated in our group for their hyphenation with CE.

The on-line combination of highly efficient CE separations and MS is now well established and offers numerous major benefits. ${ }^{[10]}$ As in many liquid-based separation techniques, electrospray ionization (ESI) is the predominant method for performance of on-line CE-MS. The advantages of ESI are simplicity, high ionization efficiency in terms of ions released from charged droplets, and ability to produce multiply charged ions. ESI is thus well suited for the analysis of ionizable or polar compounds, ideally separated by capillary zone electrophoresis, such as small pharmaceutical molecules and intact proteins. ${ }^{[11-13]}$

Atmospheric pressure photoionization (APPI) was introduced as a technique complementary to ESI to broaden the range of ionizable analytes. The coupling of APPI to CE-MS was investigated in our group because of its low ion suppression and to evaluate its sensitivity at the very low flow rates of CE separations. Numerous parameters for coupling CE to APPI-MS were optimized with a chemometric approach ${ }^{[14]}$ to simultaneously examine effects and significance of APPI parameters on method sensitivity and efficiency. In addition, a wider choice of CE electrolytes (buffer and additives) was found available for MS coupling, with limited background noise and source contamination. In this context, APPI was used for the efficient coupling of microemulsion electrokinetic chromatography (MEEKC) with MS. This approach gave good selectivity for the complex separation of ionized and neutral compounds in positive and negative ionization modes in APPI, ${ }^{[15]}$ as well as for $\log$ P determinations. ${ }^{[16]}$

In the context of macromolecule analysis, the coupling of $\mathrm{CE}$ with TOF/ MS offers a powerful tool that can be used to detect and identify proteins.[17] An efficient, rapid, and simple CE-ESITOF/MS procedure was developed for the analysis of endogenous human growth hormone $(\mathrm{hGH})$ and recombinant human growth hormone (rhGH), in collaboration with the LAD. ${ }^{[11]}$ Attention was focused on optimizing CE and ESI conditions to enhance selectivity, efficiency, and sensitivity while hindering protein adsorption onto the capillary walls. ${ }^{[18}$ To optimize CE-ESI-MS interfacing, a chemometric approach was implemented to evaluate the most relevant factors and determine the optimal conditions. A high 


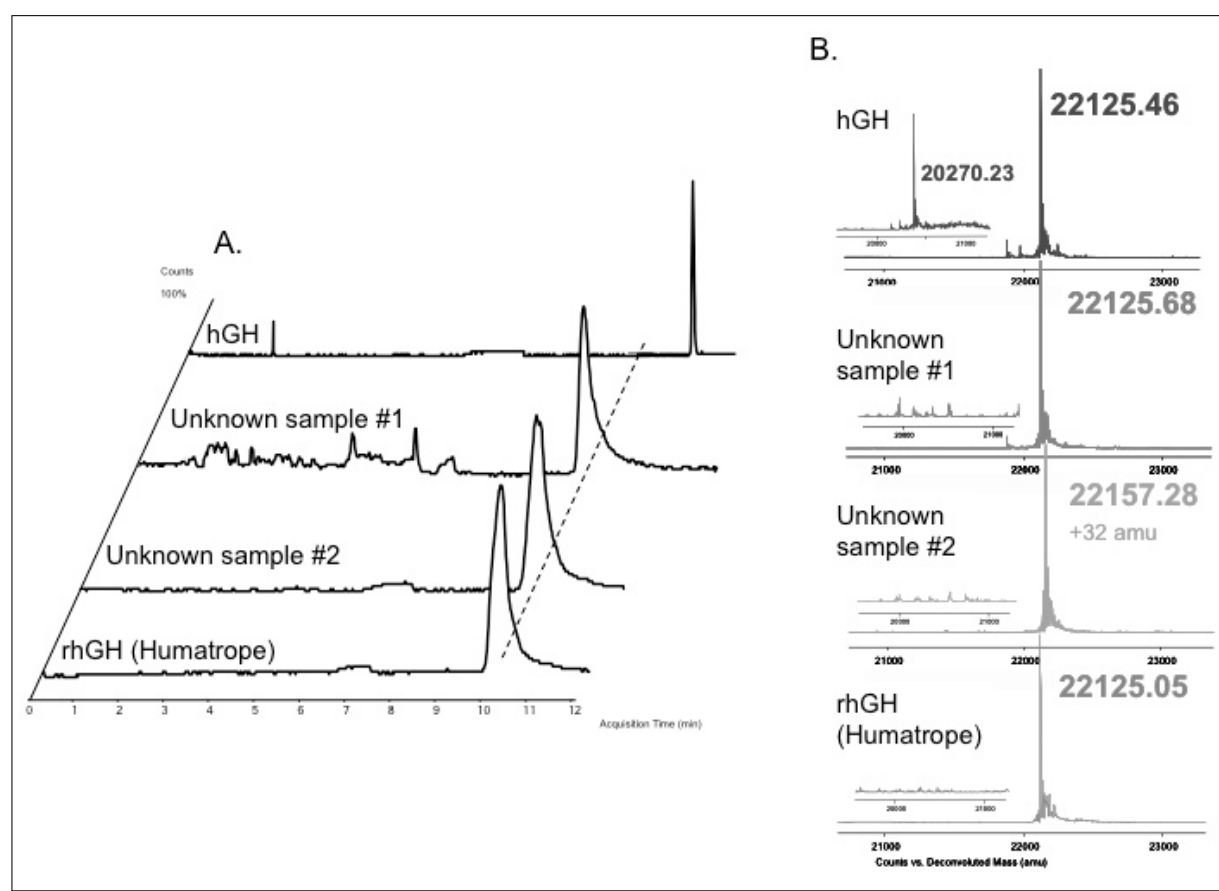

Fig. 2. Analysis of unknown samples by CE-ESI-TOF/MS. A. Electropherograms. B. Respective deconvoluted spectra. Upper trace: standard of human growth hormone (hGH) at $200 \mathrm{mg} / \mathrm{mL}$. Upper middle trace: unknown sample 1. Lower middle trace: unknown sample 2. Lower trace: recombinant hGH (Humatrope) at $200 \mathrm{mg} / \mathrm{mL}$. Adapted from ref. [11] with permission. degree of selectivity was mandatory to distinguish endogenous hGH from rhGH. After method optimization, the separation of hGH and rhGH was achieved (Fig. 2A), and the confirmation was obtained by the deconvolution of their respective mass spectra. The MS information revealed that the hGH exhibited both the 22 and $20 \mathrm{kDa}$ isoforms, whereas rhGH exhibited only the $22 \mathrm{kDa}$ isoform (Fig. 2B). This method was evaluated for the successful identification of rhGH in unknown seized samples. CE-ESI-TOF/MS was also applied in doping analysis for artificial oxygen carriers known as hemoglobin-based oxygen carriers (HBOCs), the use of which has recently emerged. ${ }^{[12]}$ In this application, the TOF analyzer was used to provide accurate mass measurements of CE peaks to confirm the presence of HBOCs. Additional selectivity was gained due to protein dissociathe ability to distinguish bovine and human hemoglobins based on the differences in their monomeric chain molecular weights. This analytical method was applied successfully to plasma samples enriched with a commercially available HBOC.

The potential of CE-ESI-TOF/MS for quantitation of intact proteins was assessed for the simultaneous characterization and quantitative analysis of insulin. ${ }^{[13]}$ A strategy based on multiple injections was selected, and the methodology was fully validated according to international guidelines such as ICH. The method was successfully applied to the analysis of insulin formulations obtained from regular tion in the ESI source, which resulted in and parallel markets. This strategy could be implemented in a regulatory context for quality control as well as for the detection of counterfeits.

The proportion of counterfeit medicines has indeed dramatically increased in the last few years. According to numerous official sources, for some pharmaceutical wholesalers in African countries, the pro- portion has reached $80 \%$. Unfortunately, the situation cannot be improved because of the lack of suitable analytical equipment. In collaboration with the College of Engineering and Architecture of Fribourg (University of Applied Sciences of Western Switzerland), we developed a low cost $\mathrm{CE}$ device equipped with a new deep UV detector based on LED technology (Fig. 3). ${ }^{[19]}$ The aim of this project is to provide CE-based methods and quality control protocols to laboratories in emerging countries for the analysis of essential medicines from a list compiled by the WHO (www.who.int/medicines). These substances are vital in the health care systems of emerging countries. Eight representative active compounds were selected by Prof. P. Bonnabry, chief pharmacist of the University Hospitals of Geneva, for their various physical-chemical properties (acidic, basic or neutral analytes, pKa, log $\mathrm{P}$, etc.), and the drug formulations were analyzed with the new CE system (Fig. 3). ${ }^{[20]}$

\section{Chemometrics \& Data Mining: Advanced Analysis and Integration of Omics Data}

The combination of MS with separative techniques such as LC or CE greatly increases the quality and the quantity of the raw data generated. By taking advantage of modern analytical platforms, high-throughput strategies have been implemented to assess the metabolic profiles of plants, biofluids and other complex samples with

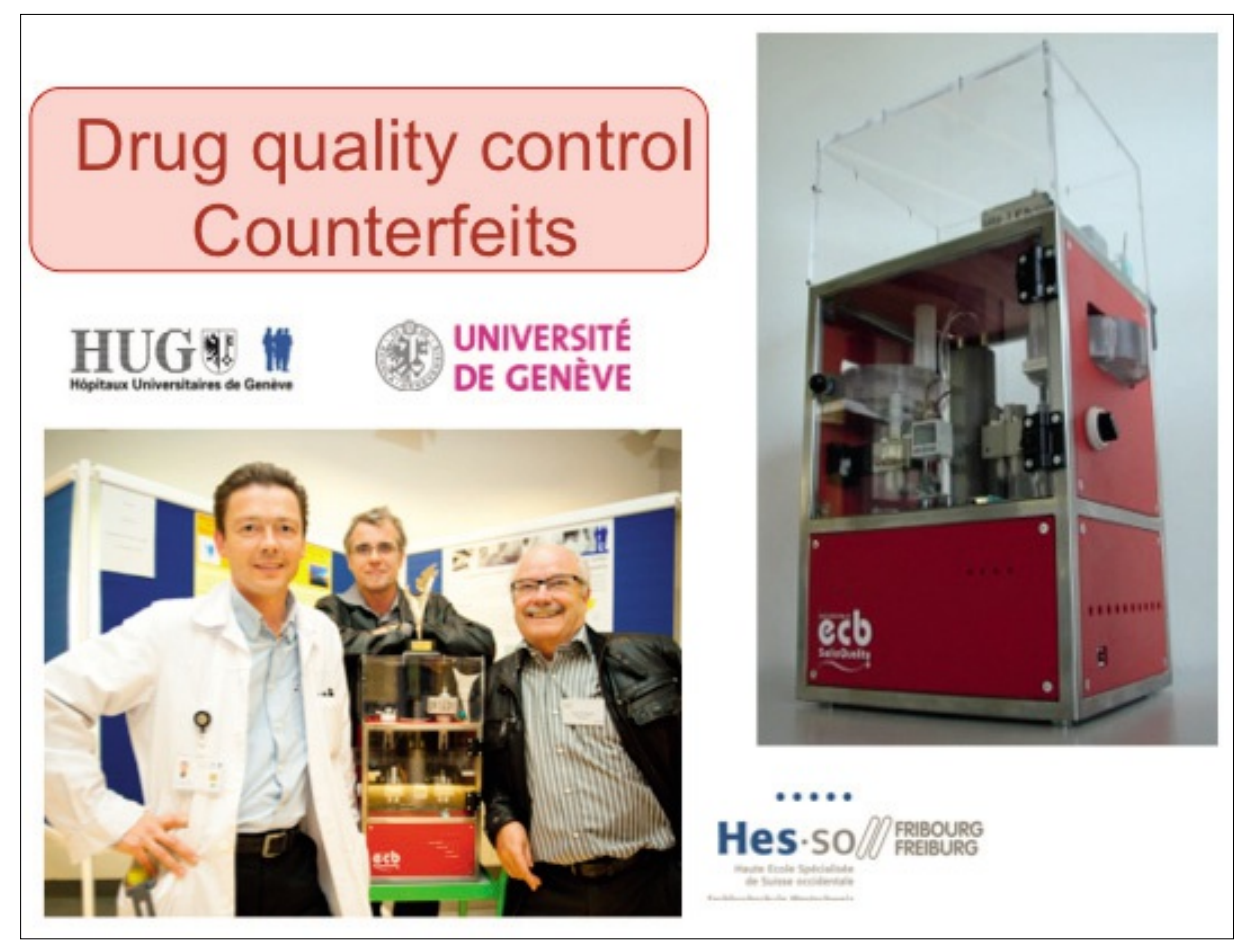

Fig. 3. Low-cost CE apparatus and academic partners at the Innovation Day of the Hospital University of Geneva - October 2012 (from left to right, Prof. P. Bonnabry / HUG, Dr. S. Rudaz / Unige, Prof. C. Rohrbasser / HES Fribourg). 
high sensitivity and selectivity. For a single sample, analyses can be performed in parallel to offer an extended coverage of the chemical diversity, as the sets of analyses offer complementary information. This recent profusion of information provided by holistic approaches allows innovative perspectives in pharmaceutical, toxicological and biomedical sciences. Untargeted analyses constitute a potent tool for the discovery of biomarkers related to a physiological response and the diagnosis of complex phenotypes. Such untargeted experiments involve interdisciplinary knowledge starting from sample collection and preparation to analytical procedures, raw data processing and data analysis.[21]

Extracting knowledge from data, revealing patterns among samples and identifying critical or discriminant variables is not a straightforward task. Chemometrics is a research field that tries to address these considerations, as it aims to process and understand complex multivariate data structures. Multivariate methodologies provide potent alternatives to classical statistical methods by summarizing the information content of a large number of potentially correlated and noisy signals into comprehensible concepts. Such models are expected to provide both robustness and easy interpretability of data of massive dimensionality by reducing the data complexity. Models based on latent variables such as principal component analysis (PCA) and projection to latent structures (PLS) constitute attractive solutions to provide compact representations of data and diagnostic tools for the detection of biomarkers. Handling and analyzing such complex data has a great impact on the quality of the identification and quantification of biomarkers and therefore to the ultimate biological interpretation.

In that perspective, several collaborative projects involving expert knowledge in analytics, chemometrics, phytochemistry and plant biology were initiated at the EPGL to highlight minor signaling biomarkers induced by mechanical wounding in model plants. An untargeted methodology involved a two-step analytical approach, combining a fast metabolite fingerprinting phase and a high resolution metabolite profiling phase. ${ }^{[2,23]}$ Chemometric and data mining tools were applied for visualization and differential analysis to survey metabolic changes related to defense signaling in the most comprehensive manner. ${ }^{[2,25]}$ In an additional study, machine learning algorithms, including classification and feature selection methods (Neural Networks, Support Vector Machines, Decision Trees, etc.), were evaluated in relationship with the intrinsic characteristics of metabolomic data (high dimensionality, high degree of correlation, biological variability). ${ }^{26]}$
As data structures become increasingly complex (multi-way tensors of high order or multi-block data tables), the development of new solutions is essential to cope with these new types of data. Recently, there has been strong interest in multi-way and multi-block methods for exploratory data mining and integration in systems biology. In partnership with the Swiss Centre for Applied Human Toxicology (SCAHT, Geneva, Switzerland), a steroidomic study was designed to monitor steroid glucuronidated or sulfated metabolites in urine based on UHPLC-QTOF-MS ${ }^{\mathrm{E}}$. The data mining methodology proposed to combine a targeted steroid profile analysis and an untargeted steroidomic approach for the extended coverage of steroid metabolites to provide a deeper insight into the urinary excretion pattern after oral testosterone intake. Advanced chemometric tools, such as N-PLS-DA and OPLS-DA, were applied to build classification models and extract the relevant biological information of clinical data structures involving the temporal monitoring of several variables in a series of individuals ${ }^{[27]}$ (Fig. 4). Other issues regarding the development and progression of several major diseases can be tackled by the monitoring of steroids. Disturbances in the steroid profile induced by xenobiotics can usually be detected as phenotypic changes, and the mechanisms underlying these changes are mostly unknown.

Finally, multi-block methodologies that take advantage of data generated through an experimental design approach also constitute promising alternatives. Such organization of the experiments with a view of subsequent analysis represents a very stimulating opportunity for the development of new holistic strategies. ${ }^{[28]}$

\section{Conclusions}

Analytical developments should improve the ability to detect a large number of compounds even at low concentrations in a complex matrix. In that respect, separation techniques such as LC/UHPLC, SFC, and $\mathrm{CE}$ constitute mandatory tools to separate analytes and allow optimal conditions for their detection. The study of fundamental aspects of separation science is essential to increase the overall resolution needed in various fields. As high-resolution MS is expected to help the identification of unknown analytes, untargeted approaches, instead of focusing on a restricted number of targeted compounds, constitute promising tools for global monitoring. These new strategies also need to be implemented by taking into account more environment-

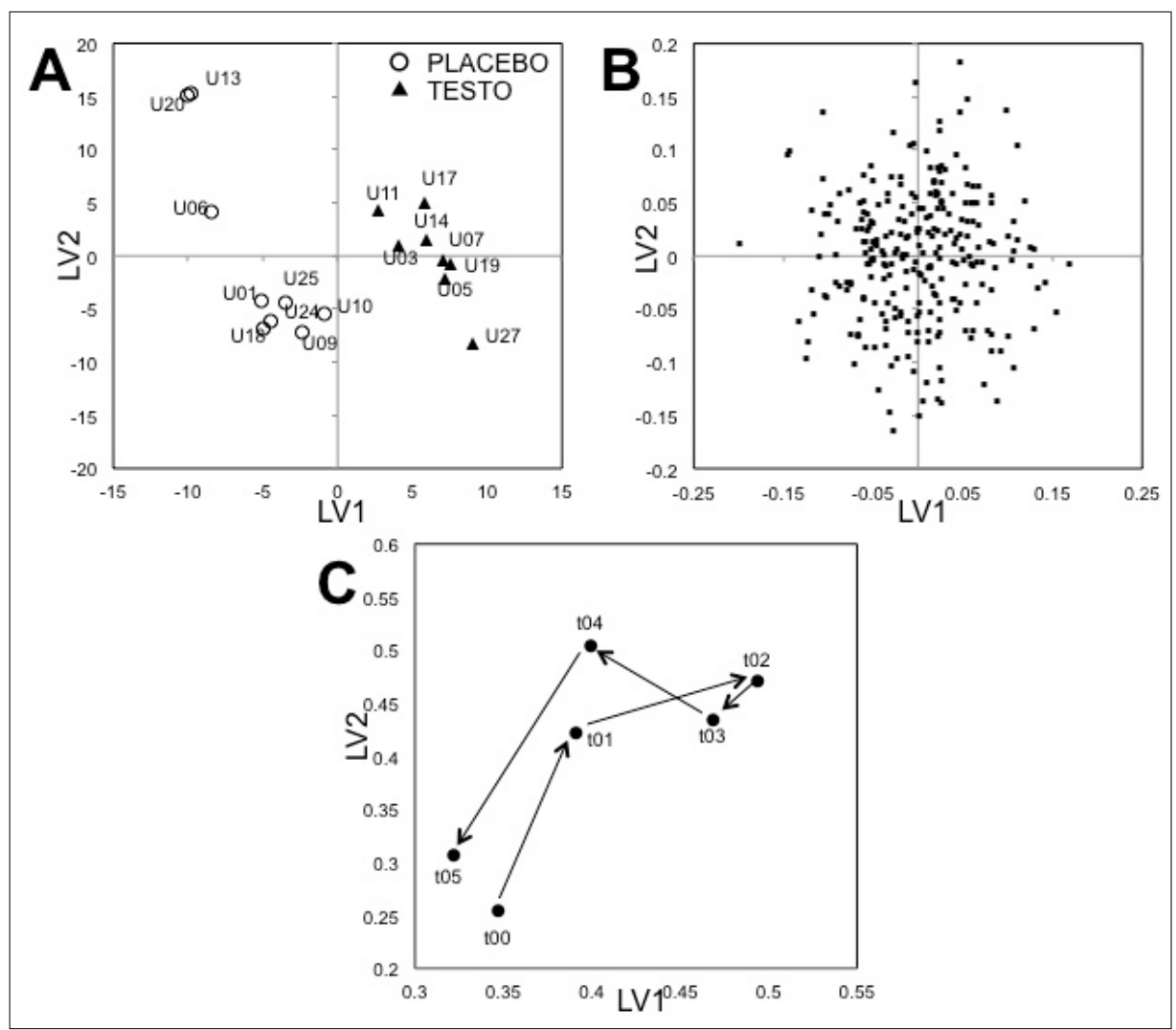

Fig. 4. N-PLS-DA model from UHPLC-TOF/MSE steroidomic profiles. A. Sample mode scatter plot, volunteers from the PLACEBO group are symbolized by white circles $(\bigcirc)$ and individuals from the TESTOSTERONE group by black triangles (A). B. Variable mode scatter plot, steroidrelated peaks are symbolized by black squares $(\boldsymbol{\square})$. C. Time mode scatter plot, time points are symbolized by black circles (๑). Adapted from ref. [27] with permission. 
friendly protocols. Automating the process of information extraction and knowledge discovery will certainly acquire a growing importance in pharmaceutical and biomedical research. In this context, it is important to understand and evaluate the intrinsic characteristics of both the data mining algorithms (e.g. robustness to outliers or correlations) and the data at hand (high dimensionality, correlations between variables, etc.).

Received: February 4, 2012

[1] http://www.unige.ch/sciences/pharm/fanal/ lcap/telechargement.htm, accessed February 2012.

[2] E. Grata, D. Guillarme, G. Glauser, J. Boccard, P. A. Carrupt, J. L. Veuthey, S. Rudaz, J. L. Wolfender, J. Chromatogr. A 2009, 1216, 5660.

[3] D. Guillarme, E. Grata, G. Glauser, J. L. Wolfender, J. L. Veuthey, S. Rudaz, J. Chromatogr. A 2009, 1216, 3232.

[4] F. Badoud, E. Grata, L. Perrenoud, M. Saugy, S. Rudaz, J. L. Veuthey, J. Chromatogr. A $\mathbf{2 0 1 0 ,}$ 1217, 4109.

[5] F. Badoud, E. Grata, L. Perrenoud, L. Avois, M. Saugy, S. Rudaz, J. L. Veuthey, J. Chromatogr. A 2009, 1216, 4423 .
[6] L. Novakova, J. L. Veuthey, D. Guillarme, J. Chromatogr. A 2011, 1218, 7971.

[7] D. Guillarme, J. Ruta, S. Rudaz, J. L. Veuthey, Anal. Bioanal. Chem. 2010, 397, 1069.

[8] J. Ruta, D. Zurlino, C. Grivel, S. Heinisch, J. L. Veuthey, D. Guillarme, J. Chromatogr. A 2011, $1228,221$.

[9] A. Staub, D. Zurlino, S. Rudaz, J. L. Veuthey, D. Guillarme, J. Chromatogr. A 2011, 1218, 8903.

[10] J. Schappler, J. L. Veuthey, S. Rudaz, Separation Sci. Technol. 2008, 9, 477.

[11] A. Staub, S. Giraud, M. Saugy, S. Rudaz, J. L. Veuthey, J. Schappler, Electrophoresis 2010, 31,388 .

[12] A. Staub, S. Rudaz, M. Saugy, J. L. Veuthey, J. Schappler, Electrophoresis 2010, 31, 1241.

[13] A. Staub, S. Rudaz, J. L. Veuthey, J. Schappler, J. Chromatogr. A 2010, 1217, 8041.

[14] J. Schappler, D. Guillarme, J. Prat, J. L. Veuthey, S. Rudaz, Electrophoresis 2007, 28, 3078.

[15] J. Schappler, D. Guillarme, S. Rudaz, J. L. Veuthey, Electrophoresis 2008, 29, 11.

[16] Y. Henchoz, S. Romand, J. Schappler, S. Rudaz, J. L. Veuthey, P. A. Carrupt, Electrophoresis 2010, 31, 952.

[17] A. Staub, J. Schappler, S. Rudaz, J. L. Veuthey, Electrophoresis 2009, 30, 1610.

[18] A. Staub, S. Comte, S. Rudaz, J. L. Veuthey, J. Schappler, Electrophoresis 2010, 31, 3326.

[19] C. Rohrbasser, D. Rhême, S. Décastel, S. Roth, M. L. A. Montes, J. L. Veuthey, S. Rudaz, Chimia 2009, 63, 890 .
[20] R. D. Marini, E. Rozet, M. L. A. Montes, C. Rohrbasser, S. Roht, D. Rhème, P. Bonnabry, J Schappler, J. L. Veuthey, P. Hubert, S. Rudaz, J. Pharm. Biomed. Anal. 2010, 53, 1278.

[21] J. Boccard, J. L. Veuthey, S. Rudaz, J. Sep. Sci. 2010, 33, 290.

[22] E. Grata, J. Boccard, G. Glauser, P. A. Carrupt, E. E. Farmer, J. L. Wolfender, S. Rudaz, J. Sep. Sci. 2007, 30, 2268.

[23] E. Grata, J. Boccard, D. Guillarme, G. Glauser, P. A. Carrupt, E. E. Farmer, J. L. Wolfender, S. Rudaz, J. Chromatogr. B 2008, 871, 261.

[24] J. Boccard, E. Grata, A. Thiocone, J. Y. Gauvrit, P. Lanteri, P. A. Carrupt, J. L. Wolfender, S. Rudaz, Chemometr. Intell. Lab. Syst. 2007, 86, 189.

[25] G. Glauser, J. Boccard, S. Rudaz, J. L. Wolfender, Phytochem. Analysis 2010, 21, 95.

[26] J. Boccard, A. Kalousis, M. Hilario, P. Lanteri, M. Hanafi, G. Mazerolles, J. L. Wolfender, P. A. Carrupt, S. Rudaz, Chemometr. Intell. Lab. Syst. 2010, 104, 20.

[27] J. Boccard, F. Badoud, E. Grata, S. Ouertani, M. Hanafi, G. Mazerolles, P. Lantéri, J. L. Veuthey, M. Saugy, S. Rudaz, Forensic Sci. Int. 2011, 213,85 .

[28] G. Mazerolles, J. Boccard, M. Hanafi, S. Rudaz, Chemometr. Intell. Lab. Syst. 2011, 106, 65. 